\title{
Issues Surrounding Antibiotic Use in Older Adults
}

\author{
Caitlin Biedron • Teena Chopra
}

Published online: 25 May 2013

(C) Springer Science+Business Media New York 2013

\begin{abstract}
By the year 2030, an estimated $20 \%$ of the United States population will be aged 65 years and older, and almost 30 million of these individuals are anticipated to have functional limitations necessitating long-term care. Older adults' increased susceptibility to nosocomial infections, combined with their often atypical presentation, results in a tendency to inappropriately prescribe antibiotics in long-term care facilities (LTCFs). Furthermore, reduced laboratory capacity in LTCFs can cause significant delays in receiving culture results and prolonged duration of empiric antimicrobial therapy. This review explores these factors and other issues contributing to the misuse and overuse of antibiotics as they relate to diagnosis, transmission, and management of infections in older adults. Inappropriate antimicrobial use has considerable consequences, including the development of multidrug resistant organisms, increasing incidence and mortality related to Clostridium difficile infection, and increasing adverse drug reactions, and therefore prompt and coordinated action is required to address this growing public health threat.
\end{abstract}

Keywords Older adults $\cdot$ Long-term care facilities · Antibiotic resistance $\cdot$ Clostridium difficile infection

\section{Introduction}

Antimicrobial resistance combined with the rapidly dwindling antimicrobial pipeline has emerged as a significant healthcare safety threat in the twenty-first century. Multidrug-resistant organisms (MDROs) within the healthcare setting are

\author{
C. Biedron \\ Wayne State University, School of Medicine, 320 E. Canfield Ave, \\ Detroit, MI 48201, USA \\ e-mail: cbiedron@med.wayne.edu \\ T. Chopra $(\bowtie)$ \\ Internal Medicine/Infectious Diseases, \\ Detroit Medical Center/Wayne State University, \\ University Health Center, 4201 St. Antoine, Suite 2B, \\ Detroit, MI 48201, USA \\ e-mail: tchopra@med.wayne.edu
}

responsible for an increasing incidence of hospital-acquired infections (HAIs), which have been linked to increases in morbidity, mortality, length of hospital stay, and healthcare costs [1•]. As the incidence of antimicrobial-resistant infections has been steadily rising, very little progress has been made in the development of new and effective antibiotics. Additionally, there is marked misuse of currently available antibiotics which further complicates the problem of growing antimicrobial resistance [2]. Of note, the population most severely affected by this conundrum is older adults, particularly those residing in long-term care facilities [3].

It is estimated that by the year 2030, $20 \%$ of the United States population will be aged 65 years and older, and will account for a $25 \%$ increase in healthcare spending [4]. The growing geriatric population is particularly susceptible to nosocomial infections because of multimorbidity, immune senescence, greater severity of illness, functional impairment, cognitive impairment, incontinence, and the presence of frequent short-term and long-term indwelling devices such as urinary catheters and feeding tubes [5••]. By 2030, almost 30 million older adults are anticipated to have functional limitations that will increase the need for long-term care. Currently, there are more than 16,000 long-term care facilities (LTCFs) in the U.S., which house approximately 1.5 million older adults [6].

Residents of LTCFs are at greater risk for infection than older adults residing in the community. Urinary tract infection, pneumonia, soft-tissue infection, gastroenteritis, and prosthetic device-associated infections are common problems among elderly LTCF residents. For example, UTI occurs at an incidence of 0.1-2.4 cases per 1,000 residentdays, and pneumonia develops among elderly LTCF residents at a rate of 1 episode per 1,000 resident-days, which is ten times greater than the rate of pneumonia among elderly individuals living in the community [6].

The increased susceptibility to nosocomial infections among older adults, combined with their often atypical presentation, often leads to heightened suspicion of infection, thereby causing healthcare workers to prescribe antibiotics more readily and often inappropriately in LTCFs $[7 \bullet \bullet]$. 
In this review, we discuss the various factors contributing to the misuse and overuse of antibiotics as they relate to diagnosis, transmission and management of infections in older adults. We then outline the consequences of inappropriate antimicrobial usage, including the development of multidrug resistant organisms (MDROs), increasing incidence and mortality related to Clostridium difficile infection (CDI), and increasing adverse drug reactions. Finally, we propose solutions and recommendations to address the growing challenge of inappropriate antimicrobial use in older adults.

\section{Diagnosis of Infection in Older Adults}

Typical signs and symptoms of infection are frequently absent in older adults. As one ages and becomes frailer, basal body temperature decreases, making it less likely that one will achieve the classic definition of fever. Therefore, different guidelines should be used for this patient population, and infection should be suspected in older adults with any of the following characteristics [6]:

A. Decline in functional status, defined as new or increasing confusion, incontinence, falling, deteriorating mobility, reduced food intake, or failure to cooperate with staff; OR

B. Fever if present, defined as $[1 \cdot]$ a single oral temperature $>100{ }^{\circ} \mathrm{F}\left(37.8^{\circ} \mathrm{C}\right)$; or [2] repeated oral temperatures $>99.5{ }^{\circ} \mathrm{F}\left(37.5{ }^{\circ} \mathrm{C}\right)$; or [3] an increase in temperature of $>2{ }^{\circ} \mathrm{F}\left(>1.1^{\circ} \mathrm{C}\right)$

Due to decreases in basal body temperature, elderly patients may have bacteremia with little or no fever. Studies reveal that older bacteremic patients have fewer signs or symptoms than younger bacteremic patients, and that clinical indicators alone are unreliable predictors of bacteremia in older patients. Additionally, in patients with pneumonia, tachypnea, not fever, is the only physical sign with a high predictive value [8].

Only $60 \%$ of older adults present with leukocytosis when an infection is present. As such, identifying leukocytosis can help confirm the presence of infection, but it is fairly nonspecific. An older adult with or without fever and an elevated white blood cell (WBC) count has a high probability of underlying bacterial infection if they have a high percentage of neutrophils or if they show an elevated total band (immature neutrophil) count. But leukocytosis may be minimal or absent even with severe infection including bacteremia [8].

In fact, leukocytosis often reflects a favorable prognosis by signaling that the patient's bone marrow is producing WBCs in response to an infectious or inflammatory process. A markedly depressed WBC count has been linked to poor prognosis in patients with pneumonia. The lack of an elevated WBC should not lessen the significance of other clinical signs and symptoms, such as purulent sputum or abdominal pain. Longitudinal changes in WBC may offer more information than a single value [8]. Furthermore, many older adults who use nonsteroidal anti-inflammatory drugs (NSAIDs) for painful musculoskeletal conditions have their inflammatory response altered, rendering leukocyte count a less reliable indicator of infection among these patients [9].

Bacteriuria is very common among LTCF residents and poses another challenge to clinicians [3]. Antibiotic use for suspected UTIs among LTCF residents is very frequent and most often inappropriate, as it is usually initiated in response to nonspecific symptoms or asymptomatic bacteriuria (ASB) [10]. Asymptomatic bacteriuria in an older adult is considered a benign condition that does not require treatment, and guidelines published by the Infectious Disease Society of America (IDSA) state that there is no measurable benefit to screen for or provide antibiotic treatment for ASB in older adult patients living in the community or in long-term care facilities [11]. Furthermore, LTCFs that used standardized diagnostic and treatment algorithms for patients with a suspected UTI were able to significantly improve appropriate use of antibiotics, leading to a decline in overall antibiotic use as compared to use in LTCFs that did not implement such algorithms [12].

\section{Challenges to Appropriate Antimicrobial Use Among Older Adults Residing in LTCFs}

Long-term care facilities have emerged as a major health care delivery system across the nation owing to the increasing demands to reduce health care costs and decrease the length of hospital stays [13]. About 1.4 million older adults now reside in skilled nursing facilities (SNFs) and over 40,000 admissions to long-term acute care hospitals (LTACs) now occur annually [14], and these numbers are expected to increase further in the years ahead due to the growing geriatric population $[5 \cdot \bullet]$.

\section{Empiric Treatment in Older Adults}

Unfortunately, determining whether an older adult patient truly needs an antimicrobial agent is often difficult for a variety of reasons, including the following:

1. The lack of typical signs and symptoms of infection in older adults further narrows the fine line between colonization and infection, leading to a higher suspicion of infection, and hence an increased use of antimicrobials [6].

2. Most LTCFs outsource their microbiological laboratory testing, causing a delay in obtaining culture results (speciation and susceptibility), which in turns leads to longer duration of empiric antimicrobial therapy [3]. 
3. Finally, the availability of physicians to evaluate LTCF residents for possible infection is also highly variable; Daneman et al. [15] found that less than $44 \%$ of residents had a physician visit within one day of their antimicrobial prescription start date. Consequently, antibiotics are often prescribed over the telephone in this setting [3].

\section{Patterns of Antibiotic Use in LTCFs}

Antibiotics are used extensively in LTCFs (7). Previous surveys of LTCF residents found an antibiotic usage point prevalence of approximately $7 \%$ to $10 \%$ [16-18], and found that between $50 \%$ and $70 \%$ of residents receive at least one course of antibiotics per year $[16,19,20]$. Although antibiotic usage varies greatly between facilities, antibiotics are generally thought to be overused in LTCFs [7••]. Various studies suggest that $25 \%$ to $75 \%$ of systemic antimicrobials are inappropriately prescribed in LTCFs [16-18]. Studies assessing prescribing practices vary in many ways, and different standards are often used to judge appropriateness, leading to variability across the literature. However, many studies now rely on surveillance criteria to assess whether the case in question has met diagnostic criteria for an infectious disease [7••]. One such study, conducted by Loeb and colleagues [21], evaluated appropriateness of antibiotic prescriptions using standardized surveillance definitions of common infections, and found that only $49 \%$ of all prescriptions for infections met diagnostic criteria.

The result of such widespread use of antimicrobials is that individuals are at risk for colonization or infection with drugresistant organisms, and higher rates of antibiotic-related complications such as Clostridium difficile colitis [7••].

\section{Transmission of Infection Among Older Adults}

\section{Infection Control Challenges in LTCFs}

Yet another factor contributing to this vicious cycle of increased antimicrobial use, increased colonization by MDROs, and increased HAIs in LTCFs is the use of inadequate infection prevention practices in many LTCFs. As LTCFs accept increasingly complex patients from acute care facilities, infection prevention becomes crucial. However, LTCFs have many unique characteristics that create special challenges in implementing an effective infection prevention program $[5 \cdot \bullet]$.

Most LTCFs are required to provide socialization of residents through group activities. While these activities are important for promoting good physical and mental health, they may also increase communicable infectious disease exposure and transmission [3]. Additionally, occupational and physical therapy activities at LTCFs, while vital to restoring or maintaining physical and mental function, may increase the risk of person-to-person transmission between residents or exposure to contaminated environmental surfaces through the use of physical or occupational therapy equipment [3].

\section{Care Transitions}

Another major challenge to appropriate antimicrobial use and the spread of multidrug resistance among older adults is encountered during care transitions from one healthcare setting to another. With the reduction in the average length of hospital stay, not only has the severity of illness among residents of LTCFs increased but so has the number of rapid transfers between facilities, during which older adults often serve as vectors, transmitting pathogens from one setting to another $[5 \bullet \bullet]$.

Such transitions are also extremely important with regards to Clostridium difficile infection (CDI), as higher rates of interfacility transmission and prevalence of Clostridium difficile infections have been identified among this patient population. Surveillance data obtained in 2010 by the Emerging Infections Program of the Centers for Disease Control and Prevention (CDC) indicates that many CDI cases occur in patients exposed to multiple settings [22•]. For instance, $20 \%$ of hospital-onset CDIs occurred in recent residents of a nursing home, and $67 \%$ of nursing home-onset CDI cases occurred in patients recently discharged from an acute care hospital [22•]. These findings underscore the fact that the risk for CDI from antibiotic exposure and transmission moves with patients across multiple health-care settings, necessitating the need for collaboration and recognition of the interdependence of health-care settings in order to reduce CDI rates [22•].

\section{Consequences of Inappropriate Antibiotic Use}

\section{Multidrug Resistant Organisms (MDROs) in LTCFs}

Infections caused by MDROs, although more common in acute care hospitals, are also increasing significantly in LTCFs [23]. The frequent use of antibiotics in LTCFs has led to the selection of a resistant flora [24, 25], and the proximity of residents and contact between residents and healthcare workers facilitates the spread of these organisms [26]. The most common MDROs found in LTCFs have been methicillin-resistant Staphylococcus aureus (MRSA) and vancomycin-resistant Enterococcus (VRE), although gramnegative organisms, such as Klebsiella and Acinetobacter, are being seen with increasing frequency $[24,27,28]$. Additionally, transmission of these MDROs to previously noncolonized residents has been observed [29]. In this setting, infection with MDROs has been associated with increased 
morbidity, mortality, and cost [30, 31]. Furthermore, LTCF residence has been frequently identified as a risk factor for antibiotic-resistant infection in hospitalized patients [3].

Elderly and disabled residents are at increased risk for colonization with resistant organisms, and colonization may persist for long periods of time, such as months to years, in such individuals $[3,32]$. Within a LTCF, length of stay in the facility and placement in rooms with multiple beds have both been identified as risk factors for transmission of MRSA [33]. Both infected and colonized residents may serve as sources of transmission of MDROs in the LTCF $[34,35]$. Furthermore, when a MDRO such as MRSA becomes endemic within a facility, unfortunately elimination is highly unlikely [35]. For these reasons, LTCFs can expect infections with MDROs to be an ongoing challenge, and therefore steps must be taken to address this threatening public health problem with a greater sense of urgency.

Unfortunately, during the last decade there has also been a dramatic drop in the development and approval of new antibacterial agents [3]. Importantly, no drugs have reached advanced stages of development for infection due to MDR Gram-negative bacilli, such as $A$. baumannii and P. aeruginosa [2]. As such, our therapeutic options for these pathogens are now so extremely limited that clinicians are forced to use older, previously discarded drugs, such as colistin, that are associated with significant toxicity and for which there is a lack of robust data to guide selection of dosage regimen or duration of therapy [36-39]. The side effects of these drugs, such as nephrotoxicity in the case of colistin, are especially dangerous in elderly patients, who often have underlying comorbidities such as chronic kidney disease, and therefore even these older agents may not provide a viable treatment option for such patients [2]. The absence of appropriate agents to treat infection due to resistant gram-negative bacilli places elderly patients with these infections in danger of poor outcomes.

\section{Clostridium Difficile Infection (CDI) in LTCFs}

The main modifiable risk factor for CDI is antimicrobial use, which increases risk through an alteration in the patient's normal lower-intestinal flora [40]. Consequently, the indiscriminate use of antibiotics in LTCFs leads to an increased risk of CDI among residents, who are already more vulnerable to $C$. difficile infection due to their increased age and other factors, as described below.

The disproportional clinical burden of CDI in older adults is related to a number of factors, including comorbidities, greater need for hospitalization or institutional care and therefore greater exposure to the pathogen; selection for strains with increased pathogenicity; immunoscenescence with poor ability to mount an adequate immune response; and greater exposure to antibiotics that suppress the colonization resistance afforded by normal gut microflora $[41 \bullet \bullet]$.
CDI is the most common cause of nosocomial diarrhea [42] and a growing problem in long-term acute care facilities and skilled nursing facilities [43]. Since 2001, CDI incidence, mortality, and recurrence rates have been increasing [44-46]. The estimated number of deaths attributable to CDI increased from 3,000 deaths per year during 1999-2000 to 14,000 during 2006-2007, with more than $90 \%$ of deaths among patients 65 years of age or older [47]. Additionally, both recurrent and severe CDI are more likely with increasing age [48].

According to the $\mathrm{CDC}$, the majority of $C$. difficile cases occur in nursing homes or other outpatient settings [22•]. Overall, $94 \%$ of all CDIs are related to healthcare exposures; of these, $75 \%$ have their onset outside of hospitals (nursing homes, outpatient settings, and recently discharged patients) [22•]. In LTCFs, the incidence of CDI may now be as high as 3.72 cases per 1,000 resident-days [49].

Such findings emphasize the need for improved CDI management practices related to diagnosis, contact isolation, and environmental disinfection to be incorporated into established infection control programs at LTCFs. Furthermore, the introduction of antimicrobial stewardship programs (ASPs) at long-term care facilities would further reduce the prevalence of CDIs among LTCF residents.

\section{Increases in Antibiotic-Related Adverse Drug Reactions}

Antibiotic-associated adverse events lead to many emergency department visits, with allergic reactions being the most common events. In 2008, there were approximately 142,000 visits to emergency departments for adverse events attributed to antibiotics [50].

Frail older adults appear to have a particularly high risk of developing adverse drug reactions. In some cases, this is because the effect of age and frailty on the elimination of the drug is not taken into account and too large a dose is therefore prescribed [51]. For instance, the pharmacokinetics of many drugs are affected by renal function and body weight. In general, older adults have a smaller body mass than younger individuals, and their kidney function is diminished by comparison. It has been demonstrated that creatinine clearance is decreased by $\sim 40 \%$ in individuals $>80$ years of age, despite serum creatinine values that appear to be normal [52]. Therefore, appropriate dose reductions for antibiotics, such as quinolones, which are eliminated primarily through the kidneys, may reduce the likelihood of exaggerated drug exposures and, thus, toxicities in this population [52]. Furthermore, minimizing unnecessary antibiotic use overall by even a small percentage through the introduction of ASPs could significantly reduce the direct risks of drug-related adverse events in elderly patients in LTCFs [50]. 


\section{Recommendations}

In the last few decades, healthcare delivery has moved from acute care facilities to skilled nursing facilities, rehabilitation units, assisted-living facilities, home, and outpatient settings. Furthermore, measures to reduce healthcare costs have led to a reduced number of hospitalizations and shorter lengths of stay, resulting in an increasing acuity of illness among LTCF residents. In addition to higher device utilization, these residents are more likely to be provided antimicrobial therapy and physical rehabilitation services than LTCF residents were in the past [53].

As LTCFs have emerged as a major healthcare delivery site, infection prevention and appropriate antibiotic use have become even more critical in these facilities. Since the environment of LTCFs provides a major reservoir for MDROs, and LTCFs house a more susceptible older adult population, controlling the spread and colonization of MDROs in such facilities is of paramount importance. However, infection control measures and antimicrobial stewardship programs have not been scaled-up in accordance with the more prominent role that these various healthcare settings now play in the provision of geriatric care [3]. The recommendations below provide strategies and approaches which can begin to address the numerous factors surrounding antibiotic use and multidrug resistance in long-term care facilities.

In order to more accurately diagnose infection in elderly patients residing in LTCFs, the initial clinical evaluation should be a three-tiered approach involving a certified nursing assistant (CNA), the on-site nurse, and a physician assistant or physician. CNAs are often the first to recognize a symptom or sign of infection in LTCF residents, but data suggest that they frequently misinterpret these initial clinical signs and symptoms. Therefore, after measuring the patient's vital signs, the CNA should report to the on-site nurse immediately if residents are suspected of having an infection or have a fever. The clinical evaluation can then be continued by the nurse and eventually by another clinician, allowing a more accurate diagnosis to be made, thereby facilitating more judicious use of antibiotics [6]. Additionally, because the inappropriate treatment of asymptomatic bacteriuria has also been identified as a significant cause of antibiotic overuse in LTCFs, this must be a major target for antimicrobial stewardship programs in this setting.

As occupational and physical therapy programs are commonly provided at LTCFs, a specific infection control program for rehabilitation services should be developed and should focus on facility design in order to promote hand hygiene compliance, including easy access to sinks and the use of alcohol-based hand sanitizer for all residents and therapists participating in rehabilitation sessions. Furthermore, patients who are infectious should not be treated at the central physical therapy or rehabilitation facility $[5 \bullet \cdot$.
Care transitions present another challenge to infection control prevention and appropriate antibiotic use at LTCFs. It is therefore important for the receiving facility to request clinical information from the transferring facility regarding current culture reports for residents who may be infected or colonized with pathogenic organisms, especially multi-drug resistant organisms. This action would enable healthcare workers at the receiving facility to determine the interventions necessary to meet the resident's needs and to prevent the spread of pathogens within the facility $[5 \bullet \bullet]$. Furthermore, detailed information regarding any previous antimicrobial regimens should be provided to the new facility, including dosage and duration, so that continued administration can be evaluated upon admission to the new facility and the care team can decide whether the current regimen should be continued, modified, or discontinued at the new facility.

Finally, ASPs focused on timely de-escalation and discontinuation of antimicrobials are urgently needed in LTCFs in order to facilitate proper prescribing practices among HCWs caring for elderly patients, which will in turn decrease colonization pressure by multidrug resistant organisms. Implementation of ASPs has the potential to limit antimicrobial resistance in LTCFs, while improving treatment efficacy, minimizing drug-related adverse events, and reducing treatment-related costs $[7 \bullet \bullet]$.

\section{Conclusions/Expert Opinion}

Antibiotic stewardship and infection control are inextricably linked, and both must be strengthened in the long-term care setting. If ASPs are not properly implemented, and inappropriate antibiotic use continues, the spread of MDROs will increase and greater infection rates will be observed in elderly patients living in LTCFs. Conversely, if poor infection control practices are implemented in LTCFs, an increase in infection rates will result, which in turn will necessitate an increase in antibiotic use and cause higher rates of MDROs.

Stewardship programs must be implemented in all types of long-term care facilities, with an emphasis on appropriate use of antibiotics in older adult patients and within the longterm care environment. A focus on de-escalation is critical in such care settings, and ASPs provide a structured way for HCWs to become better informed about the indications for antibiotics and more compliant with recommended regimen stop dates. Furthermore, facilities can tailor the CDC's 12 -step campaign to prevent antimicrobial resistance among long-term care residents to their unique patient population and facility needs $[10,54]$.

Infection control programs within LTCFs must place a greater emphasis on safe and effective transitions of care 
between facilities, especially as residents of LTCFs have become more critically ill, and often require transport between acute care facilities and LTCFs on a more regular basis. Communication between facilities is essential during such transitions, and care liaisons can help immensely at such critical junctions in care. The incorporation of CDI management practices is also of utmost importance, as rates of severe CDI have begun to increase among elderly patients living at LTCFs. In addition to these aspects, the standard elements of an effective IC program, including hand hygiene, isolation precautions, and outbreak management, must be strengthened as well $[5 \cdot \bullet]$. The prominent role of the infection control professional is also critical to the success and effectiveness of such programs.

In order to make such changes feasible, facilities should invest in hiring physician directors who have received training in both infection control and antibiotic stewardship principles and implementation. Furthermore, geriatric and infectious disease fellows should receive more comprehensive training in appropriate antibiotic use in older adults earlier in their training.

\section{Compliance with Ethics Guidelines}

Conflict of Interest Caitlin Biedron is supported by a scholarship from the Infectious Diseases Society of America (IDSA).

Teena Chopra has received speakers' honoraria from Optimer, Pfizer, Merck, and Forest, and is supported by grants from the National Institutes of Health (NIH) and Optimer.

Human and Animal Rights and Informed Consent This article does not contain any studies with human or animal subjects performed by any of the authors.

\section{References}

Papers of particular interest, published recently, have been highlighted as:

- Of importance

•- Of major importance

1. - Spellberg B, Blaser M, Guidos RJ, Boucher HW, Bradley JS, Eisenstein BI, et al. Combating antimicrobial resistance: policy recommendations to save lives. Clin Infect Dis. 2011;52 Suppl 5:S397428. This policy paper summarizes the Infectious Disease Society of America's (IDSA) recommendations in terms of addressing the simultaneous crises of rising rates of antibiotic resistance and waning approvals of new antibiotics. IDSA puts forth public policy strategies and research activities that have the potential to preserve the effectiveness of antibiotics and reverse the concerning decline in the antibiotic $R \& D$ pipeline.

2. Boucher HW, Talbot GH, Bradley JS, Edwards JE, Gilbert D, Rice LB, et al. Bad bugs, no drugs: no ESKAPE! An update from the Infectious Diseases Society of America. Clin Infect Dis. 2009; 48(1):1-12.
3. Smith PW, Bennett G, Bradley S, Drinka P, Lautenbach E, Marx J, et al. SHEA/APIC guideline: infection prevention and control in the long-term care facility. Am J Infect Control. 2008;36(7):50435 .

4. Dubberke ER, Reske KA, Olsen MA, McDonald LC, Fraser VJ. Short- and long-term attributable costs of Clostridium difficileassociated disease in nonsurgical inpatients. Clin Infect Dis. 2008;46(4):497-504.

5. • Flanagan E, Chopra T, Mody L. Infection prevention in alternative health care settings. Infect Dis Clin N Am. 2011;25(1):27183. This article outlines the key elements of an infection control program in a skilled nursing facility, which should include surveillance for infections and antimicrobial resistance, an outbreak investigation and control plan for epidemics, isolation precautions, and hand hygiene. It also highlights the unique aspects of LTCF residents which make infection control efforts more challenging, including their need for rehabilitation and physical therapy services and their frequent involvement in transitions of care.

6. High KP, Bradley SF, Gravenstein S, Mehr DR, Quagliarello VJ, Richards $\mathrm{C}$, et al. Clinical practice guideline for the evaluation of fever and infection in older adult residents of long-term care facilities: 2008 update by the Infectious Diseases Society of America. Clin Infect Dis. 2009;48(2):149-71.

7. - Smith PW, Watkins K, Miller H, et al. Antibiotic stewardship programs in long-term care facilities. Ann Long-Term Care Clin Care Aging. 2011;19(4):20-5. This article provides an overview of antibiotic use and resistance in long-term care facilities, and discusses how various components of antibiotic stewardship programs can be applied to the long-term care setting. Furthermore, a step-wise approach to implementation of an ASP is recommended, starting with the least costly and intrusive steps, and gradually working towards introducing an antimicrobial appropriateness review process, which requires expert review of individual cases.

8. Levenson S. Fever and leukocytosis. Caring Ages. 2003;4(7):1547.

9. Amella EJ. Presentation of illness in older adults. Am J Nurs. 2004;104(10):40-51. quiz 2.

10. Michigan Antibiotic Resistance Reduction (MARR) Coalition. Long-term care toolkit. [Accessed April 2013]; Available from: http://mi-marr.org/LTC toolkit.php.

11. Gross PA, Patel B. Reducing antibiotic overuse: a call for a national performance measure for not treating asymptomatic bacteriuria. Clin Infect Dis. 2007;45(10):1335-7.

12. Loeb M, Brazil K, Lohfeld L, McGeer A, Simor A, Stevenson K, et al. Effect of a multifaceted intervention on number of antimicrobial prescriptions for suspected urinary tract infections in residents of nursing homes: cluster randomised controlled trial. BMJ. 2005;331(7518):669.

13. Friedman C, Barnette M, Buck AS, Ham R, Harris JA, Hoffman P, et al. Requirements for infrastructure and essential activities of infection control and epidemiology in out-of-hospital settings: a consensus panel report. Association for Professionals in Infection Control and Epidemiology and Society for Healthcare Epidemiology of America. Infect Control Hosp Epidemiol Consens Dev Conf. 1999;20(10):695-705.

14. Kahn JM, Benson NM, Appleby D, Carson SS, Iwashyna TJ. Long-term acute care hospital utilization after critical illness. JAMA. 2010;303(22):2253-9.

15. Daneman N, Gruneir A, Newman A, Fischer HD, Bronskill SE, Rochon PA, et al. Antibiotic use in long-term care facilities. J Antimicrob Chemother. 2011;66(12):2856-63.

16. Nicolle LE, Bentley DW, Garibaldi R, Neuhaus EG, Smith PW. Antimicrobial use in long-term-care facilities. SHEA long-termcare committee. Infect Control Hosp Epidemiol. 2000;21(8):537-45. 
17. Warren JW, Palumbo FB, Fitterman L, Speedie SM. Incidence and characteristics of antibiotic use in aged nursing home patients. J Am Geriatr Soc. 1991;39(10):963-72.

18. Zimmer JG, Bentley DW, Valenti WM, Watson NM. Systemic antibiotic use in nursing homes. A quality assessment. J Am Geriatr Soc. 1986;34(10):703-10.

19. Mylotte JM. Measuring antibiotic use in a long-term care facility. Am J Infect Control. 1996;24(3):174-9.

20. Lee YL, Thrupp LD, Friis RH, Fine M, Maleki P, Cesario TC. Nosocomial infection and antibiotic utilization in geriatric patients: a pilot prospective surveillance program in skilled nursing facilities. Gerontology. 1992;38(4):223-32.

21. Loeb M, Simor AE, Landry L, Walter S, McArthur M, Duffy J, et al. Antibiotic use in Ontario facilities that provide chronic care. J Gen Intern Med. 2001;16(6):376-83.

22. - Vital signs: preventing Clostridium difficile infections. MMWR Morb Mortal Wkly Rep. 2012;61(9):157-62. This report demonstrates that of all health-care-associated CDIs, $75 \%$ have their onset outside of hospitals, and argues that major reductions will therefore require antibiotic stewardship along with infection control applied to long-term care and ambulatory-care settings as well as hospitals. It also advocates for the extension of State health department programs to cover healthcare settings beyond hospitals, where they have already demonstrated success in preventing CDIs.

23. Crnich CJ, Safdar N, Robinson J, Zimmerman D. Longitudinal trends in antibiotic resistance in US nursing homes, 2000-2004. Infect Control Hosp Epidemiol. 2007;28(8):1006-8.

24. Siegel JD, Rhinehart E, Jackson M, Chiarello L. Management of multidrug-resistant organisms in health care settings, 2006. Am J Infect Control. 2007;35(10 Suppl 2):S165-93.

25. Siegel JD, Rhinehart E, Jackson M, Chiarello L. 2007 Guideline for isolation precautions: preventing transmission of infectious agents in health care settings. Am J Infect Control. 2007;35(10 Suppl 2):S65-164.

26. O'Fallon E, Harper J, Shaw S, Lynfield R. Antibiotic and infection tracking in Minnesota long-term care facilities. J Am Geriatr Soc. 2007;55(8):1243-7.

27. Sengstock DM, Thyagarajan R, Apalara J, Mira A, Chopra T, Kaye KS. Multidrug-resistant Acinetobacter baumannii: an emerging pathogen among older adults in community hospitals and nursing homes. Clin Infect Dis. 2010;50(12):1611-6.

28. Bonomo RA. Multiple antibiotic-resistant bacteria in long-termcare facilities: an emerging problem in the practice of infectious diseases. Clin Infect Dis. 2000;31(6):1414-22.

29. O'Fallon E, Schreiber R, Kandel R, D'Agata EM. Multidrugresistant gram-negative bacteria at a long-term care facility: assessment of residents, healthcare workers, and inanimate surfaces. Infect Control Hosp Epidemiol. 2009;30(12):1172-9.

30. Suetens C, Niclaes L, Jans B, Verhaegen J, Schuermans A, Van Eldere J, et al. Methicillin-resistant Staphylococcus aureus colonization is associated with higher mortality in nursing home residents with impaired cognitive status. J Am Geriatr Soc. 2006; 54(12):1854-60.

31. Capitano B, Nicolau DP. Evolving epidemiology and cost of resistance to antimicrobial agents in long-term care facilities. $\mathrm{J}$ Am Med Dir Assoc. 2003;4(3 Suppl):S90-9.

32. Boyce JM. Methicillin-resistant Staphylococcus aureus in hospitals and long-term care facilities: microbiology, epidemiology, and preventive measures. Infect Control Hosp Epidemiol. 1992; 13(12):725-37.

33. Wendt C, Svoboda D, Schmidt C, Bock-Hensley O, von Baum H. Characteristics that promote transmission of Staphylococcus aureus nursing homes in German nursing homes. Infect Control Hosp Epidemiol. 2005;26(10):816-21.

34. Flournoy DJ. Antimicrobial susceptibilities of bacteria from nursing home residents in Oklahoma. Gerontology. 1994;40(1):53-6.
35. Boyce JM, Jackson MM, Pugliese G, Batt MD, Fleming D, Garner JS, et al. Methicillin-resistant Staphylococcus aureus (MRSA): a briefing for acute care hospitals and nursing facilities. The AHA technical panel on infections within hospitals. Infect Control Hosp Epidemiol. 1994;15(2):105-15.

36. ArftN S. National Nosocomial Infections Surveillance (NNIS) system report, data summary from January 1992 through June 2004, issued October 2004. Am J Infect Control. 2004;32(8): 470-85.

37. Falagas ME, Bliziotis IA. Pandrug-resistant Gram-negative bacteria: the dawn of the post-antibiotic era? Int J Antimicrob Agents. 2007;29(6):630-6.

38. Urban C, Bradford PA, Tuckman M, Segal-Maurer S, Wehbeh W, Grenner L, et al. Carbapenem-resistant escherichia coli harboring klebsiella pneumoniae carbapenemase $\beta$-lactamases associated with long-term care facilities. Clin Infect Dis. 2008;46(11): e127-30

39. Falagas ME, Kasiakou SK, Saravolatz LD. Colistin: the revival of Polymyxins for the management of multidrugresistant gram-negative bacterial infections. Clin Infect Dis. 2005;40(9):1333-41.

40. Cohen SH, Gerding DN, Johnson S, Kelly CP, Loo VG, McDonald LC, et al. Clinical practice guidelines for Clostridium difficile infection in adults: 2010 update by the society for healthcare epidemiology of America (SHEA) and the infectious diseases society of America (IDSA). Infect Control Hosp Epidemiol. 2010;31(5):431-55.

41. • Louie TJ, Miller MA, Crook DW, Lentnek A, Bernard L, High $\mathrm{KP}$, et al. Effect of age on treatment outcomes in Clostridium difficile infection. J Am Geriatr Soc. 2013;61(2):222-30. This article demonstrates that risk factors accounting for poorer outcomes of Clostridium difficile treatment with advancing age include infection with the B1 strain type, inpatient status, renal insufficiency, leukocytosis, hypoalbuminemia, and concomitant medication exposure. It also argues that the measurable deterioration in treatment outcomes that occurred with advancing age underscores the need for more effective prevention and treatment strategies.

42. Kelly CP. A 76-year-old man with recurrent Clostridium difficileassociated diarrhea: review of C. difficile infection. JAMA. 2009;301(9):954-62.

43. Kelly CP, LaMont JT. Clostridium difficile-more difficult than ever. N Engl J Med. 2008;359(18):1932-40.

44. Blossom DB, McDonald LC. The challenges posed by reemerging Clostridium difficile infection. Clin Infect Dis Off Publ Infect Dis Soc Am. 2007;45(2):222-7.

45. Loo VG, Poirier L, Miller MA, Oughton M, Libman MD, Michaud $\mathrm{S}$, et al. A predominantly clonal multi-institutional outbreak of Clostridium difficile-associated diarrhea with high morbidity and mortality. N Engl J Med. 2005;353(23):2442-9.

46. Pepin J, Alary ME, Valiquette L, Raiche E, Ruel J, Fulop K, et al. Increasing risk of relapse after treatment of Clostridium difficile colitis in Quebec, Canada. Clinical Infect Dis Off Publ Infect Dis Soc Am. 2005;40(11):1591-7.

47. Hall AJ, Curns AT, McDonald LC, Parashar UD, Lopman BA. The roles of Clostridium difficile and norovirus among gastroenteritisassociated deaths in the United States, 1999-2007. Clin Infect Dis. 2012;55(2):216-23.

48. Miller M, Gravel D, Mulvey M, Taylor G, Boyd D, Simor A, et al. Health care-associated Clostridium difficile infection in Canada: patient age and infecting strain type are highly predictive of severe outcome and mortality. Clin Infect Dis. 2010; 50(2):194-201.

49. Laffan AM, Bellantoni MF, Greenough 3rd WB, Zenilman JM. Burden of Clostridium difficile-associated diarrhea in a long-term care facility. J Am Geriatr Soc. 2006;54(7):1068-73. 
50. Shehab N, Patel PR, Srinivasan A, Budnitz DS. Emergency department visits for antibiotic-associated adverse events. Clin Infect Dis. 2008;47(6):735-43.

51. Routledge PA, O'Mahony MS, Woodhouse KW. Adverse drug reactions in elderly patients. Br J Clin Pharmacol. 2004; 57(2):121-6.

52. Owens RC, Ambrose PG. Antimicrobial safety: focus on fluoroquinolones. Clin Infect Dis. 2005;41(Supplement 2):S144-57.
53. Richards Jr CL, Darradji M, Weinberg A, Ouslander JG. Antimicrobial use in post-acute care: a retrospective descriptive analysis in seven long-term care facilities in Georgia. J Am Med Dir Assoc. 2005;6(2):109-12.

54. Centers for Disease Control and Prevention. Antibiotic use in nursing homes. [Accessed April 2013]; Available from: http:// www.cdc.gov/getsmart/healthcare/learn-from-others/factsheets/ nursing-homes.html. 